\title{
RANDOM OSCILLATION IN SYSTEMS SUBJECT TO THE EXCITATION OF A CLASS OF COLOURED NOISES
}

\author{
NGUYEN DONG ANH \\ Institute of Mechanics, $\mathrm{Ha}$ Noi
}

\begin{abstract}
SUMMARY. In the paper the higher order stochastic averaging method is developed to a class of coloured noise excitations when the corresponding forming filter has only finite negative eigenvalues. The second approximate probability density function to the Duffing system subject to the exponentially correlated process is obtained. Numerical application is carried out to investigate the independence of mean square amplitude on the bandwidth parameter
\end{abstract}

\section{INTRODUCTION}

Nonlinear random vibrations in dynamical systems subjected to the excitation of a white noise have often been investigated by many authors. It is wellknown that the white noise process has a constant spectral density function and thus does not exits in the practice. Mean white, the random processes with linear-fractional spectral density functions of the frequency describe well many real environmental loadings. These processes contain an important class of coloured noises which can be interpreted as the result of the passage of white noises through a certain linear system with constant parameters, called a forming filter. In the paper the excitation of a class of coloured noises on a single degree of freedom system is investigated.

\section{A TYPE OF FORMING FILTER}

Consider a mechanical system whose motion is described in the form

$$
\ddot{x}+\omega^{2} x=\varepsilon f(x, \dot{x})+\sqrt{\varepsilon} q(t) .
$$

The excitation $q(x)$ is a normal stationary random process, the result of the passage of a white noise through the linear forming filter

$$
L q(t)=\frac{d^{n}}{d t^{n}} q(t)+\sum_{s=0}^{n-1} \alpha_{s} \frac{d^{s}}{d t^{s}} q(t)=b_{\zeta}(t)
$$

where $\alpha_{s}, b=$ const, $\varsigma(t)$ in a white noise of unit intensity. In Eq. (2.1), $\omega$ is the natural frequency, $\epsilon$ is a positive small parameter, $f(x, \dot{x})$ is a nonlinear function of displacement and velocity. The spectral density of the process $q(t)$ can be easy obtained from (2.2)

$$
S_{q}(\omega)=\frac{1}{2 \pi} \frac{b^{2}}{\ell(i \omega) \ell(-i \omega)}
$$

where

$$
\ell(\lambda)=\lambda^{n}+\sum_{s=0}^{n-1} \alpha_{s} \lambda^{\prime}
$$


Consider a case when the linear filter (2.2) has only finite negative eigenvalue, i.e., all the roots $\lambda_{i}$ of the characteristic equation

$$
\ell(\lambda)=0
$$

are distinct, real, negative, and

$$
|\lambda|>\varepsilon
$$

Eliminating now $q(t)$ from (2.1), (2.2) one obtains

$$
L\left(\ddot{x}+\omega^{2} x\right)=\varepsilon L f(x, \dot{x})+\sqrt{\varepsilon} b \dot{\zeta}(t)
$$

Forming the characteristic equation of the generating linear equation $(\varepsilon=0)$

$$
\left(\lambda^{2}+\omega^{2}\right) \ell(\lambda)=0
$$

one gets the general solution in the form

$$
x(t)=\sum_{i=1}^{n} C_{i} e^{\lambda_{i} t}+a \cos \varphi, \quad \varphi=\omega t+\theta
$$

Thus, according to the averaging method we make the replacement [4]

$$
\frac{d^{k} x}{d t^{k}}=a(t) \frac{\partial^{k}}{\partial t^{k}} \cos (\nu t+\theta(t)) \quad k=0,1, \ldots, n+1
$$

where $a(t), \theta(t)$ are Markov diffusion processes satisfying the equations

$$
\begin{aligned}
& \dot{a}=\varepsilon u_{1}(a, \theta)+\sqrt{\varepsilon} \nu_{1}(a, \theta) \dot{\zeta}(t) \\
& \dot{\theta}=\varepsilon u_{2}(a, \theta)+\sqrt{\varepsilon} \nu_{2}(a, \theta) \dot{\zeta}(t)
\end{aligned}
$$

or turning to the variable $a, \varphi$ one gets

$$
\begin{aligned}
& \dot{a}=\varepsilon u_{1}(a, \varphi)+\sqrt{\varepsilon} \nu_{1}(a, \varphi) \dot{\zeta}(t) \\
& \dot{\varphi}=\omega+\varepsilon u_{2}(a, \varphi)+\sqrt{\varepsilon \nu_{2}}(a, \varphi) \dot{\zeta}(t)
\end{aligned}
$$

where one has

$$
\begin{aligned}
& u_{1}(a, \varphi)=-\frac{1}{\omega r}[L f(x, \dot{x})]_{0} \sin \left(\varphi+\varphi_{*}\right)+\frac{b^{2}}{2 a \omega^{2} r^{2}} \cos ^{2}\left(\varphi+\varphi_{*}\right) \\
& u_{2}(a, \varphi)=-\frac{1}{a \omega r}[L f(x, \dot{x})]_{0} \cos \left(\varphi+\varphi_{0}\right)-\frac{b^{2}}{a^{2} \dot{\omega}^{2} r^{2}} \cos \left(\varphi+\varphi_{*}\right) \sin \left(\varphi+\varphi_{*}\right) \\
& \nu_{1}(a, \varphi)=\frac{b}{\omega r} \sin \left(\varphi+\varphi_{*}\right), \quad \nu_{2}(a, \varphi)=\frac{b}{a \omega r} \cos \left(\varphi+\varphi_{*}\right) \\
& {[L f(x, \dot{x})]_{0}=L f(x, \dot{x}) \frac{d x^{k}}{d t^{k}}=a \frac{\partial^{k}}{\partial t^{k}} \cos \varphi} \\
& \varphi_{*}=\sum_{i=1}^{n} \varphi_{i}, \quad r=\prod_{i=1}^{n} r_{i} \\
& r_{i}=\sqrt{\lambda_{i}^{2}+\omega^{2}}, \quad \sin \varphi_{i}=\frac{\omega}{r_{i}}, \quad \cos \varphi_{i}=-\frac{\lambda_{i}}{r_{i}} \\
& D_{i}=\prod_{m>i>0}^{n}\left(\lambda_{m}-\lambda_{i}\right)\left(\lambda_{i}-\lambda_{s}\right)
\end{aligned}
$$


For system (2.12), the corresponding Fokker-Planck equation for stationary probability density function $W(a, \varphi)$ of amplitude and phase can be formed as follows

$$
\omega \frac{\partial W}{\partial \varphi}=-\varepsilon L_{1}(W)
$$

where the operator $L_{1}$ is defined as

$$
L_{1}(W)=\frac{\partial}{\partial a}\left(u_{1} W\right)-\frac{1}{2} \frac{\partial^{2}}{\partial a^{2}}\left(\nu_{1}^{2} W\right)+\frac{\partial}{\partial \varphi}\left[u_{2} W-\frac{\partial}{\partial a}\left(\nu_{1} \nu_{2} W\right)-\frac{1}{2} \frac{\partial}{\partial \varphi}\left(\nu_{2}^{2} W\right)\right]
$$

The solution $W(a, \varphi)$ of Eq. (2.14) is to be obtained in the form [5]

$$
W(a, \varphi)=\sum_{i=0}^{\infty} \varepsilon^{i} W_{i}(a, \varphi)
$$

Substituting (2.16) into (2.14) and comparing the coefficients of like powers of $\varepsilon$ gives

$$
\begin{gathered}
\omega \frac{\partial W_{0}}{\partial \varphi}=0 \\
\omega \frac{\partial W_{i+1}}{\partial \varphi}=-L\left(W_{i}\right), \quad i=0,1,2, \ldots
\end{gathered}
$$

Since the functions $W_{i}(a, \varphi)$ must be periodic ones of variable $\varphi$, one gets from $(2.18)$

$$
\left\langle L_{i}\left(W_{i}\right)\right\rangle=0
$$

where $(>$ is defined as the averaging operator

$$
\langle.\rangle=\frac{1}{2 \pi} \int_{0}^{2 \pi}(.) d \varphi
$$

Thus, one gets from (2.17) and (2.19) for $i=0$

$$
\begin{gathered}
W_{0}=W_{0}(a) \\
\left\langle L\left(W_{0}(a)\right)\right\rangle=0
\end{gathered}
$$

We expand into a series of Fourier

$$
\begin{aligned}
u_{i}(a, \varphi) & =\sum_{j=0}^{m}\left(S_{i j}(a) \sin 2 j \varphi+C_{i j}(a) \cos 2 j \varphi\right) \\
\nu_{\ell}(a, \varphi) \nu_{k}(a, \varphi) & =\sum_{j=0}^{m}\left(S_{\ell k}(a) \sin 2 j \varphi+C_{\ell k j}(a) \cos 2 j \varphi\right), \quad i, \ell, k=1,2
\end{aligned}
$$

Substituting (2.22) into $(2.15)$ yield

$$
\begin{aligned}
L\left(W_{0}(a)\right)= & \sum_{j=0}^{m}\left\{\left[\frac{\partial}{\partial a}\left(S_{1 j} W_{0}\right)-\frac{1}{2} \frac{\partial^{2}}{\partial a^{2}}\left(S_{11 j} W_{0}\right)\right] \sin 2 j \varphi\right. \\
& +\left[\frac{\partial}{\partial a}\left(C_{1 j} W_{0}-\frac{1}{2} \frac{\partial^{2}}{\partial a^{2}}\left(C_{11 j} W_{0}\right)\right] \cos 2 j \varphi+\frac{\partial}{\partial \varphi}\left[\left(S_{2 j} W_{0}-\frac{\partial}{\partial a}\left(S_{12 j} W_{0}\right)\right.\right.\right. \\
& \left.\left.\left.+j W_{0} C_{22 j}\right) \sin 2 j \varphi+\left(C_{2 j} W_{0}-\frac{\partial}{\partial a}\left(C_{12 j} W_{0}\right)-j W_{0} S_{22 j}\right) \cos 2 j \varphi\right]\right\}
\end{aligned}
$$


Substituting (2.23) into (2.21) gives the equation for $W_{0}(a)$

$$
\frac{\partial}{\partial a}\left(C_{10} W_{0}\right)-\frac{1}{2} \frac{\partial^{2}}{\partial a^{2}}\left(C_{110} W_{0}\right)=0
$$

After finding $W_{0}(a)$ and substituting it into $(2.18)$ for $i=0$ one gets

$$
W_{1}(a, \varphi)=-\frac{1}{\omega} \int L\left(W_{0}\right) d \varphi
$$

or noting (2.23)

$$
W_{1}(a, \varphi)=W_{0}(a) W_{10}(a)+\sum_{j=1}^{m}\left(W_{j \diamond}(a) \sin 2 j \varphi+W_{j c}(a) \cos 2 j \varphi\right)
$$

where it is denoted

$$
\begin{aligned}
& W_{j,}(a)=-\frac{1}{\omega}\left\{\frac{1}{2 j}\left[\frac{\partial}{\partial a}\left(C_{1 j} W_{0}\right)-\frac{1}{2} \frac{\partial^{2}}{\partial a^{2}}\left(C_{11 j} W_{0}\right)\right]+S_{2 j} W_{0}-\frac{\partial}{\partial a}\left(S_{12 j} W_{0}\right)+j W_{0} C_{22 j}\right\} \\
& W_{j c}(a)=-\frac{1}{\omega}\left\{C_{2 j} W_{0}-\frac{\partial}{\partial a}\left(C_{12 j} W_{0}\right)-j S_{22 j} W_{0}-\frac{1}{2}\left[\frac{\partial}{\partial a}\left(S_{1 j} W_{0}\right)-\frac{1}{2} \frac{\partial^{2}}{\partial a^{2}}\left(S_{11 j} W_{0}\right)\right]\right\}
\end{aligned}
$$

The arbitrary integration function $W_{10}(a)$ in (2.26) will be defined by considering (2.18) and (2.19) for the case $i=1$

$$
\begin{gathered}
\omega \frac{\partial W_{2}}{\partial \varphi}=-L\left(W_{1}\right) \\
\left\langle L\left(W_{1}\right)\right\rangle=0
\end{gathered}
$$

Substituting (2.26) into (2.29) yields the differential equation for $W_{10}(a)$

$\left(C_{10} W_{0} W_{10}\right)-\frac{1}{2} \frac{\partial}{\partial a}\left(C_{110} W_{0} W_{10}\right)+\frac{1}{2} \sum_{j=0}^{m}\left\{\left(S_{1 j} W_{j \rho}+C_{1 j} W_{j c}\right)-\frac{1}{2} \frac{\partial}{\partial a}\left(S_{11 j} W_{j o}+C_{11 j} W_{j c}\right)\right\}=0$

Finally, using (2.24) one obtains the explicit form of $W_{10}(a)$

$$
\begin{aligned}
W_{10}(a)= & \sum_{j=1}^{m}\left\{\int \frac { 1 } { C _ { 1 0 } ( a ) W _ { 0 } ( a ) } \left[S_{1 j}(a) W_{j s}(a)+C_{1 j}(a) W_{j c}(a)-\frac{1}{2} \frac{\partial}{\partial a}\left(S_{11 j}(a) W_{j s}(a)\right.\right.\right. \\
& \left.\left.\left.+C_{11 j}(a) W_{j c}(a)\right)\right] d a\right\}
\end{aligned}
$$

where the coefficients $C_{1 k}(a), S_{1 k}(a), C_{1 k j}, S_{1 k j}, W_{0}(a), W_{j s}(a), W_{j c}(a)$ are found from (2.22), (2.24) and (2.27), respectively. Thus, finally according to the averaging procedure the second approximate solution to the FP equation (2.14) is defined as

$$
W(a, \varphi)=W_{0}(a)+\varepsilon\left[W_{0}(a) W_{10}(a)+W_{11}(a, \varphi)\right]
$$

So, it is important that the second approximate solution can be obtained in explicit form as it is shown from (2.26), (2.27) and (2.31).

\section{APPLICATION TO THE EXPONENTIALLY CORRELATED PROCESS}

Let $q(t)$ be an exponentially correlated stationary random process, with following spectral density and correlation function 


$$
S_{q}(\omega)=\frac{\delta_{1}^{2}}{\pi} \frac{a}{a^{2}+\omega^{2}}, \quad K_{y}(\tau)=\delta_{1}^{2} e^{-\alpha|\tau|}, \quad \sigma \gg \varepsilon
$$

The corresponding forming filter is

$$
L q=\dot{q}(t)+\alpha q(t)=\delta_{1} \sqrt{2 \alpha} s(t)
$$

According to the procedure described the solution of the system $(2.1) ;(3.2)$ is defined in the form

$$
x(t)=a \cos \varphi, \quad \dot{x}=-a \omega \sin \varphi, \quad \ddot{x}=-a \omega^{2} \cos \varphi
$$

For the case (3.2) one has, see (2.13)

$$
\begin{aligned}
& n=1, \quad \lambda=-a, \quad \sin \varphi_{1}=\frac{\omega}{\sqrt{\alpha^{2}+\omega^{2}}}, \quad \cos \varphi_{1}=\frac{\alpha}{\sqrt{\alpha^{2}+\omega^{2}}} \\
& \varphi_{*}=\varphi_{1}, \quad r=r_{1}=\sqrt{\alpha^{2}+\omega^{2}} \\
& L f=\frac{d f}{d t}+\alpha f
\end{aligned}
$$

The amplitude and phase differential equations are described by (2.12) where

$$
\begin{aligned}
& u_{1}(a, \varphi)=-\frac{(d f / d t+\alpha f)_{0} \sin \left(\varphi+\varphi_{1}\right)}{\omega \sqrt{\alpha^{2}+\omega^{2}}}+\frac{\delta_{1}^{2} \alpha^{2} \cos ^{2}\left(\varphi+\varphi_{1}\right)}{\omega^{2} a\left(\omega^{2}+\alpha^{2}\right)} \\
& u_{2}(a, \varphi)=-\frac{(d f / d t+\alpha f)_{0} \cos \left(\varphi+\varphi_{1}\right)}{\alpha \omega \sqrt{\alpha^{2}+\omega^{2}}}-\frac{\delta_{1}^{2} \alpha^{2} \sin ^{2}\left(\varphi+\varphi_{1}\right)}{\omega^{2} a^{2}\left(\omega^{2}+\alpha^{2}\right)} \\
& \nu_{1}(a, \varphi)=\frac{\delta_{1} \sqrt{2 \alpha} \sin \left(\varphi+\varphi_{1}\right)}{\omega \sqrt{\alpha^{2}+\omega^{2}}}, \quad \nu_{2}(a, \varphi)=\frac{\delta_{1} \sqrt{2 \alpha} \cos \left(\varphi+\varphi_{1}\right)}{\omega a \sqrt{\alpha^{2}+\omega^{2}}}
\end{aligned}
$$

Further, the solution to the corresponding FP equation can be performed as described above

\section{DUFFING OSCILLATOR}

It is wellknown that the exact solution of the Duffing oscillator subject to coloured noise it not available up to now. So, the approximate solutions are to be interested. So, consider the Duffing system

$$
\bar{x}+2 \varepsilon h \dot{x}+\omega^{2} x+\varepsilon \gamma x^{2}=\sqrt{\varepsilon} q(t)
$$

where $q(t)$ is the exponentially correlated random process (3.1). For this case, one gets

$$
f(x, \dot{x})=-2 h \dot{x}-\gamma x^{3}, \quad \frac{d f}{d t}+\alpha f=-2 h \ddot{x}-2 \alpha h \dot{x}-3 \gamma x^{2} \dot{x}-\alpha \gamma x^{\dot{3}}
$$

Substituting (3.3), (3.4), (4.2) into (3.5) gives

$$
\begin{aligned}
u_{i}(a, \varphi) & =\sum_{j=0}^{2}\left(S_{i j}(a) \sin 2 j \varphi+C_{i j}(a) \cos 2 j \varphi\right) \\
\nu_{\ell}(a, \varphi) \nu_{k}(a, \varphi) & =\sum_{j=0}^{\ell}\left(S_{\ell k j}(a) \sin 2 j \varphi+C_{\ell k j}(a) \cos 2 j \varphi\right)
\end{aligned}
$$


where:

$$
\begin{aligned}
& r^{2}=a^{2}+\omega^{2} \\
& S_{10}(a)=0, \quad C_{10}(a)=-h a+\frac{\delta_{1}^{2} \alpha}{2 a \omega^{2} r^{2}}, \\
& S_{11}(a)=\frac{1}{\omega r^{2}}\left[\frac{\gamma a^{3}}{4}\left(\alpha^{2}-3 \omega^{2}\right)-2 \alpha h a \omega^{2}-\frac{\delta_{1}^{2} \alpha^{2}}{a r^{2}}\right] \\
& S_{12}(a)=S_{12}(a)=\left[\frac{\gamma a^{3}}{8 \omega r^{2}}\left(\alpha^{2}-3 \omega^{2}\right)\right], \quad C_{12}(a)=\frac{\alpha \gamma a^{3}}{2 r^{2}}, \\
& S_{20}(a)=0, \quad C_{20}(a)=\frac{3}{8 \omega} \gamma a^{3}, \\
& S_{21}(a)=\frac{1}{r^{2}}\left[h\left(\omega^{2}-\alpha^{2}\right)-\alpha \gamma a^{2}-\frac{\delta_{1}^{2} \alpha\left(\alpha^{2}-\omega^{2}\right)}{a^{2} \omega^{2} r^{2}}\right], \\
& C_{21}(a)=\frac{1}{r^{2}}\left[\frac{\alpha^{2} \gamma a^{2}}{2 \omega}-2 \alpha h \omega-\frac{2 \delta_{1}^{2} \alpha^{2}}{a^{2} \omega r^{2}}\right] \\
& S_{22}(a)=-\frac{\alpha \gamma a^{2}}{2 r^{2}}, \quad C_{22}(a)=\frac{\gamma a^{2}}{8 \omega r^{2}}\left(\alpha^{2}+3 \omega^{2}\right), \\
& S_{110}(a)=0, \quad C_{110}(a)=\frac{\delta_{1}^{2} \alpha}{\omega^{2} r^{2}}, \\
& S_{111}(a)=\frac{2 \delta_{1}^{2} a^{2}}{\omega r^{4}}, \quad C_{111}(a)=\frac{\delta_{1}^{2} \alpha\left(\omega^{2}-\alpha^{2}\right)}{\omega^{2} r^{4}}, \quad S_{120}(a)=0, \quad C_{120}(a)=0, \\
& S_{121}(a)=\frac{\delta_{1}^{2} \alpha\left(\alpha^{2}-\omega^{2}\right)}{a \omega^{2} r^{4}}, \quad C_{121}(a)=\frac{2 \delta_{1}^{2} \alpha^{2}}{a \omega r^{4}}, \quad S_{220}(a)=0, \quad C_{220}(a)=\frac{\delta_{1}^{2} \alpha}{a^{2} \omega^{2} r^{2}}, \\
& S_{221}(a)=-\frac{2 \delta_{1}^{2} \alpha^{2}}{a^{2} \omega r^{4}}, \quad C_{221}(a)=\frac{\delta_{1}^{2} \alpha\left(\alpha^{2}-\omega^{2}\right)}{a^{2} \omega^{2} r^{4}}, \quad
\end{aligned}
$$

Substituting $C_{10}, C_{110}$ from (4.4) into (2.24) yields ( $C=$ const)

$$
W_{0}(a)=C a \exp \left\{-\frac{r^{2} \omega^{2} h a^{2}}{\left(\delta_{1}^{2} \alpha\right)}\right\}
$$

Further, substituting (4.5), (4.4) into (2.27) one gets

$$
\begin{array}{ll}
W_{1 s}=W_{0}(a)\left(\frac{\gamma h \omega}{2 \delta_{1}^{2}} a^{4}\right), & W_{1 \mathrm{c}}=W_{0}(a)\left(-\frac{3 \gamma}{2 r^{2}} a^{2}-\frac{\gamma h\left(\alpha^{2}-3 \omega^{2}\right)}{4 \delta_{1}^{2} \alpha} a^{4}\right) \\
W_{2 s}=W_{0}(a)\left(\frac{\gamma h \omega}{4 \delta_{1}^{2}} a^{4}\right), & W_{2 c}=W_{0}(a)\left(-\frac{3 \gamma}{4 r^{2}} a^{2}-\frac{\gamma h\left(\alpha^{2}-3 \omega^{2}\right)}{16 \delta_{1}^{2} \alpha} a^{4}\right)
\end{array}
$$

Using (2.31) for $m=2$ and noting (4.4), (4.6) after some calculations one has

$$
W_{10}(a)=-\frac{3 \gamma}{16 \delta_{1}^{2} r^{2}}\left[\gamma \omega^{2} a^{6}+\left(4 \alpha h \omega^{2}+h \alpha^{3}-\frac{h \omega^{4}}{\alpha}\right) a^{4}-\frac{6}{r^{2}} \delta_{1}^{2}\left(\omega^{2}-\alpha^{2}\right) a^{2}\right]
$$

Thus, the second approximate probability density function of amplitude and phase to the Duffing system (4.1) is found as

$$
\begin{aligned}
W(a, \varphi)= & C a \exp \left\{\frac{-\left(\alpha^{2}+\omega^{2}\right) \omega^{2} h a^{2}}{\delta_{1}^{2} \alpha}\right\}\left\{1-\frac{3 \gamma \varepsilon}{16 \delta_{1}^{2}\left(\alpha^{2}+\omega^{2}\right)}\left[\gamma \omega^{2} a^{6}+\left(4 \alpha h \omega^{2}+h \alpha^{3}-\frac{h \omega^{4}}{\alpha}\right) a^{4}\right.\right. \\
& \left.+\frac{6 \delta_{1}^{2}\left(\alpha^{2}-\omega^{2}\right) a^{2}}{\left(\alpha^{2}+\omega^{2}\right)}\right]+\frac{\varepsilon \gamma h \omega}{2 \delta_{1}^{2}} a^{4} \sin 2 \varphi-\left[\frac{3 \varepsilon \gamma}{2\left(\alpha^{2}+\omega^{2}\right)} a^{2}+\frac{\varepsilon \gamma h\left(\alpha^{2}-3 \omega^{2}\right)}{4 \delta_{1}^{2}} a^{4}\right] \cos 2 \varphi \\
& \left.+\frac{\varepsilon \gamma h \omega}{2 \delta_{1}^{2}} a^{4} \sin 4 \varphi-\left[\frac{3 \varepsilon \gamma}{4\left(\alpha^{2}+\omega^{2}\right)} a^{2}+\frac{3 \varepsilon \gamma h\left(\alpha^{2}-3 \omega^{2}\right)}{16 \delta_{1}^{2} \alpha} a^{4}\right] \cos 4 \varphi\right\}
\end{aligned}
$$


In the limit case when the coloured noise $q(t)$ tends to an white noise $\delta \xi(t)$, i.e.

$$
\alpha, \delta_{1} \rightarrow+\infty, \quad \frac{2 \delta_{1}^{2}}{\alpha} \rightarrow \delta^{2}=\text { const }
$$

the solution (4.8) tends to the following expression

$$
W(a, \varphi)=C a \exp \left\{-\frac{2 \omega^{2} h a^{2}}{\delta^{2}}\right\}\left[1-\frac{\varepsilon \gamma h}{8 \delta^{2}} a^{4}(3+4 \cos 2 \varphi+\cos 4 \varphi)\right]
$$

which is obtained in [5].

Table 1. Mean - square amplitude of Duffing oscillatior to coloured noise for $\omega=1 ; \gamma=0.1 ; h=1 ; \varepsilon=0.1 ; \sigma_{1}^{2}=20$

\begin{tabular}{rrr}
$\alpha$ & $\left\langle a^{2}\right\rangle$ & $\left\langle a^{2}\right\rangle_{0}$ \\
\hline 2.0 & 7.134 & 8.148 \\
4.9 & 4.500 & 4.719 \\
6.9 & 3.156 & 3.246 \\
8.0 & 2.143 & 2.462 \\
10.0 & 1.950 & 1.981 \\
\hline
\end{tabular}

In Tab. 1 the mean - square amplitudes of Duffing oscillator subject to coloured noise (4.1) are given when the bandwidth parameter $\alpha$ varies. It is seen that the mean - square amplitude decreases when $\alpha$ increases from 2.0 to 10.0 , and for $\alpha=10.0$ the mean - square amplitude is closed to the one (value 1.944) of the corresponding Duffing oscillator subject to the white noise with intensity equal to 0.4 . In Tab. 1 the mean - square amplitude of corresponding linear system $\left\langle a^{2}\right\rangle_{0},(y=0)$, is also given. It is shown that in the case of coloured noise the effect of cubic non-linearity can be investigated by using higher order averaging procedure proposed.

\section{CONCLUSION}

The averaging method is an useful tool for investigation both deterministic and stochastic quasilinear system. In the stochastic problems, however, the method has often been developed only for white noise excitations. The results obtained show that the higher order averaging method can be successfully extended to the cases of coloured noise excitation.

Acknowledgement. This publication is completed with financial support from the National Basic Research Program in Natural Science.

\section{REFERENCE}

1. Mitropolskii Iu. A. Averaging method in nonlinear mechanics. Naukova-Dumka, Kiev, 1971 (in Russian).

2. Stratonovich R., Topics in the theory of random noise. Gordon \& Breach v. 1, N. Y. 1963.

3. Gikhman I., Skorokhod A. Stochastic differential equations and apllications. Naukova-Dumka, Kiev, 1982 (in Russian).

4. Mitropolskii Iu. A., Dao N. V., Anh N. D. Nonlinear oscillations in systems of arbitrary order, Naukova-Dumka, Kiev, 1992 (in Russian).

5. Nguyen Dong Anh. Higher approximate solutions in stochastic averaging method. Proc. of NCSR of VN, vol. 5, 101 (1993), pp, 19-26. 